\title{
Impact of the wetting length on flexible blade spreading
}

\author{
Marion Krapez, ${ }^{1}$ Anaïs Gauthier, ${ }^{1}$ Hamid Kellay, ${ }^{2}$ Jean-Baptiste \\ Boitte, ${ }^{3}$ Odile Aubrun, ${ }^{3}$ Jean-François Joanny, ${ }^{4,5}$ and Annie Colin ${ }^{6,1}$ \\ ${ }^{1}$ MIE - Chemistry, Biology and Innovation (CBI) UMR8231, ESPCI Paris, \\ CNRS, PSL Research University, 10 rue Vauquelin, Paris, France \\ ${ }^{2}$ Laboratoire Ondes et Matière d'Aquitaine, UMR 5798, CNRS, \\ Université de Bordeaux, 351 Cours de la Libération, 33405 Talence, France \\ ${ }^{3}$ Centre de recherche de l'Oréal, 188 Rue Paul Hochart, 94550 Chevilly-Larue \\ ${ }^{4}$ Physico Chimie Curie, Institut Curie, PSL University, 26 rue d'Ulm, 75005 Paris \\ ${ }^{5}$ Collège de France, 11 place Marcelin Berthelot, 75005 Paris, France \\ ${ }^{6}$ Université de Bordeaux, Centre de recherche Paul Pascal, CNRS UMR 5031, 115 avenue Schweitzer,
}

(Dated: March 2, 2021)

\begin{abstract}
We study the spreading of a Newtonian fluid by a deformable blade, a common industrial problem, characteristic of elasto-hydrodynamic situations. Here, we consider the case of a finite reservoir of liquid, emptying as the liquid is spread. We evidence the role of a central variable: the wetting length $l_{\mathrm{w}}$, which sets a boundary between the wet and dry parts of the blade. We show that the deposited film thickness $e$ depends quadratically with $l_{\mathrm{w}}$. We study this problem experimentally and numerically by integration of the elasto-hydrodynamic equations, and finally propose a scaling law model to explain how $l_{\mathrm{w}}$ influences the spreading dynamics.
\end{abstract}

PACS numbers:

The flapping of flags or the deformation of boat sails are emblematic of the coupling of flexible sheets with a fluid flow. Beyond these large-scale situations, there is a blooming interest for smaller systems, in which the forces responsible for the deformation of the solid are viscous instead of inertial. Such situations are encountered when a fluid is confined by an elastic plate, for example paper sheets gliding on thin air just above the floor after falling from a table [1].

The spreading or the scraping of liquids with a flexible blade (Fig 1) is another paradigmatic example of this class of fluid-structure problems, with a rich non-linear behavior [1-3]. Flexible blade spreading is central in numerous industrial processes such as paper coating, which inspired early studies [4-8]. More recently, this problem has been studied in the light of an elasticity-capillarity analogy [9] and compared with another well-known system, dip coating $[10,11]$. Following this approach, the elastic forces induced by the local curvature of the sheet replace surface tension forces [12-14]. An elastic LandauLevich approach $[12,13]$ can be used to predict the film thickness as a function of the blade properties. This method, which successfully modeled selected experiments [12] is based on two assumptions: i) the blade is fully covered with liquid (which amounts to neglecting capillarity) and $i$ ) while in motion, it is only deformed over a length $l_{x}$ close to the tip. This internal length is analogous to the dynamical meniscus length in dip-coating. However, in a large majority of everyday situations, such as the spreading of a paint on a wall, or cream on the skin, the liquid reservoir is finite and the blade partially wet. This introduces a new length scale, $l_{\mathrm{w}}$ (as shown in Fig. 1), and its existence challenges the Landau-Levich approach. In this paper, we evidence the central role of $l_{\mathrm{w}}$ on the spreading dynamics, and we analyze the similarities and differences with dip-coating.

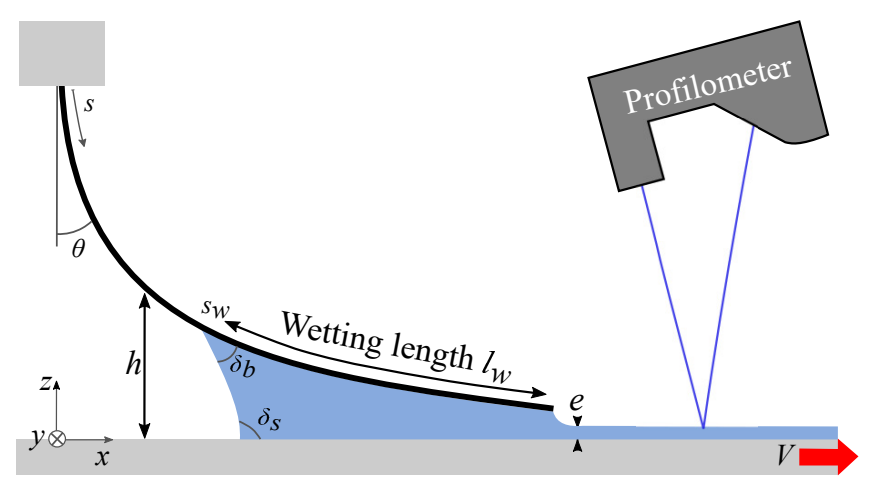

Figure 1: Experimental setup. A finite amount of fluid deposited under an elastic blade is emptied by moving the substrate at a constant speed $V$. The thickness of the deposited layer $e$ is measured by a profilometer. As the reservoir empties, the wetting length $l_{\mathrm{w}}$ diminishes, which impacts the deposit.

A Newtonian fluid (here silicone oil) with viscosity $\eta$ ranging from 480 to $960 \mathrm{mPas}$ is spread on an horizontal smooth PMMA plate by a soft blade made of PET plastic (Mylar) (Fig.1). The dynamic contact angles of oil on both surfaces are denoted by $\delta_{s}$ and $\delta_{b}$ : in a typical experiment, $\delta_{s} \simeq 100 \pm 10^{\circ}$, and $\delta_{b} \simeq 15 \pm 10^{\circ}$. The blade is cut in a rectangular shape, with a constant width $b=$ $4 \mathrm{~cm}$, a length $L$ of typically $6 \mathrm{~cm}$ and thickness $u=125$ or $250 \mu \mathrm{m}$. The upper part of the blade is clamped perpendicularly to the surface, at a height $H=0.46 \mathrm{~L}$. This clamping height ensures that the free edge of the blade is exactly tangent to the surface in absence of liquid. It 
is also the position for which the film thickness is the largest [12]. The rigidity $B$ of the blade is determined by measuring its deflection under its own weight [15]. $B$ is typically varied by a factor 10 when changing the blade thickness, with $B=1.0 \pm 0.1 \mathrm{mN} \mathrm{m}$ for $u=125 \mu \mathrm{m}$ and $B=7.1 \pm 0.5 \mathrm{mNm}$ for $u=250 \mu \mathrm{m}$. Using $B=\frac{E^{*} u^{3}}{12}$, we obtain a modified Young Modulus $E^{*}$ of the material, that already includes the Poisson ratio correction. Experimentally, $E^{*} \simeq 6.2 \mathrm{GPa}$, a value in good agreement with what is expected for type A mylar [16].

A known volume of liquid $\Omega_{0}$ (between 0.1 and 1.5 $\mathrm{mL}$ ) is deposited under the blade and then spread with a one-way movement, by moving the substrate at a constant speed $V(2.5<V<10 \mathrm{~mm} / \mathrm{s})$. The thickness of the scraped liquid film $e$ is measured with an optical profilometer (Keyence LJ-V7060K) positioned above the film, 2 millimeters from the edge of the blade (Fig. 1). A laser sheet of length $16 \mathrm{~mm}$ (in the $y$ direction) is projected onto the film, from which the film thickness $e(y, t)$ is measured as a function of time $t$ and position $y$. The liquid reservoir below the blade slowly empties as oil is scraped over a distance of $\simeq 10 \mathrm{~cm}$. One important variable here is the length of the blade in contact with the liquid, which we call the wetting length $l_{\mathrm{w}}$ (as visible in Fig.1). During an experiment, $l_{\mathrm{w}}(t)$ varies with time and typically diminishes by $2 \mathrm{~cm}$ as the reservoir empties. It is measured from the side and from the top using two optical cameras, at 10 frames per second. The camera and the profilometer are synchronized using an in-house Labview program, so that $e$ and $l_{\mathrm{w}}$ are recorded simultaneously during the spreading. The initial time $t=0$ corresponds to the setting in motion of the horizontal stage.

In Fig. 2a, the thickness $e$ of a silicone oil film (with viscosity $\eta=960 \mathrm{mPas}$ ) is mapped as a function of time $t$ and position $y$ ( $y=0$ corresponding to the center of the film). The color code varies from $e \simeq 0$ (dark blue) up to $e=400 \mu \mathrm{m}$ (bright yellow). Despite a small residual noise due to multiple light reflections, the film thickness is relatively uniform in the y-direction along the width of the blade: edge effects are only visible $2 \mathrm{~mm}$ from the edges, as shown in Supp. Fig. 1. It represents only $10 \%$ of the blade width. However, the film exhibits very large variations with time. This is even more striking when looking at the mean thickness $e(t)$, plotted in white in Fig. 2a. The film profile exhibits a sharp increase in the first 6 seconds, where the thickness grows from 0 to $230 \mu \mathrm{m}$. This corresponds to a transient state, associated with the setting in motion of the liquid below the blade, which was previously observed in similar systems [12]. However, the second part of the plot (corresponding to the steady state) strongly differs from previous experiments. We observe here a continuous reduction of the film thickness $e$ with time $t$ - from 230 to $80 \mu \mathrm{m}$ between 7 $\mathrm{s}$ and $20 \mathrm{~s}$. The decrease of $e$ with time is largest with the
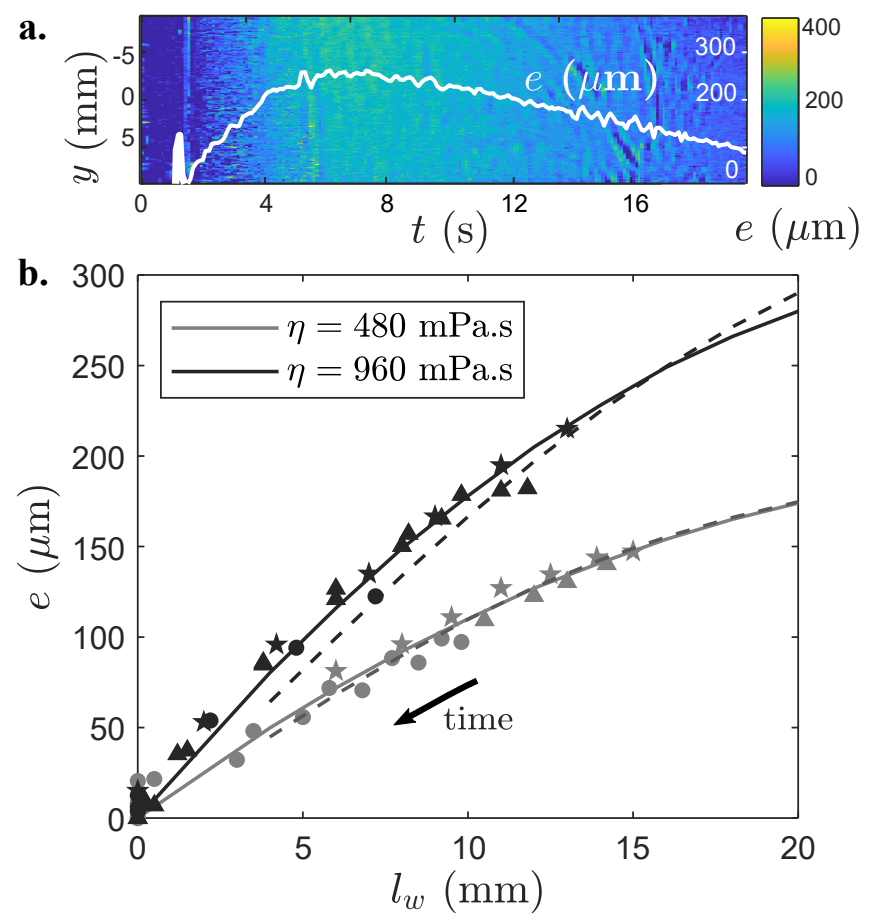

Figure 2: a. Map of the central part of the film (with viscosity $\eta=480 \mathrm{mPa}$ s) during the spreading, as a function of the width $y$ and time $t$. The color code varies from $e \simeq 0$ (dark blue) up to $e=400 \mu \mathrm{m}$ (bright yellow). The white line shows the mean thickness $e(t)$ over the film width, as a function of time. b. Film thickness $e(t)$ as a function of the wetting length $l_{\mathrm{w}}$ at the same time $t$, for two different oils with viscosity $\eta=480 \mathrm{mPa}$ s (gray) and $\eta=960 \mathrm{mPa}$ $\mathrm{s}$ (black). The markers correspond to different initial fluid volumes $\Omega_{0}: \bullet: \Omega_{0}=0.21 \pm 0.01 \mathrm{~cm}^{3}, \boldsymbol{\Delta}: \Omega_{0}=0.50 \pm 0.03$ $\mathrm{cm}^{3}$ and $\star: \Omega_{0}=0.60 \pm 0.02 \mathrm{~cm}^{3}$. The continuous lines show the scaling law (Eq.4) with prefactors 0.15 (for $\eta=480 \mathrm{mPa}$ s) and 0.17 (for $\eta=960 \mathrm{mPa} \mathrm{s}$ ) corresponding to the best fits. The dashed lines are the numerical solutions. In both experiments, $V=5 \mathrm{~mm} / \mathrm{s}, B=1.0 \mathrm{mN} \mathrm{m}$ and $L=5.7 \mathrm{~cm}$.

softest blade and the more viscous fluids, and is observed for both silicone oil and glycerine. We interpret this as a consequence of the finite reservoir size. Indeed, as the liquid empties, the length $l_{\mathrm{w}}(t)$ of the blade effectively wet by the liquid diminishes, which in turn impacts the film thickness $e(t)$. This intimate relation between $e(t)$ and $l_{\mathrm{w}}(t)$ is evidenced in Fig. 2b, where $e\left(l_{\mathrm{w}}\right)$ is measured for two oils of viscosity $\eta=480 \mathrm{mPas}$ (gray circles) and $\eta=960 \mathrm{mPas}$ (black triangles). Data points are taken at a given time $t$, so that $e$ and $l_{\mathrm{w}}$ are treated quasistatically. The markers indicate the initial liquid volume $\Omega_{0}$, varied by a factor 3 . With $\eta$ fixed, the data for all $\Omega_{0}$ overlap, which indicates that the film thickness only depends on the actual volume of the reservoir at the time $t$-a quantity measured by $l_{\mathrm{w}}(t)$. As shown in Fig. 2, the relation between $e$ and $l_{\mathrm{w}}$ is non-linear: $e$ increases more slowly for larger $l_{\mathrm{w}}$. In addition, a strong dependency of $e$ with the fluid viscosity $\eta$ is observed: $e$ increases by 
roughly $50 \%$ from $\eta=480 \mathrm{mPa}$ s to $\eta=960 \mathrm{mPas}$.

To understand how the reservoir size influences the spreading dynamics, we perform a full numerical study of the experiment. Since $e$ and $l_{\mathrm{w}}$ evolve quasi-statically with time, we solve the hydroelastic equations statically, with a constant $l_{\mathrm{w}}$ as an input parameter. The geometry is split in two zones: i) the blade itself, which interacts with the fluid below over a length $l_{\mathrm{w}}(<L)$ and ii) the free liquid film, spread by the blade. In the following, the lubricated blade shape and the free liquid film are modeled by two different sets of equations (Eq. 1 and Eq. 2), coupled by boundary conditions at the tip of the blade. In both cases, the Reynolds number $R e$ in the liquid is small, $R e=\frac{\rho e V}{\eta}<10^{-3}$, and the oil is highly confined, with $l_{\mathrm{w}} \gg e$, which allow us to use the lubrication approximation to describe the flow.

The blade shape is described as a large deflection problem, using Euler's elastica [17, 18]. In the steady state, the bending torque (expressed as a function of the curvilinear length $s$ along the blade) is equal to the torque $\Gamma$ produced by the external forces, which arise from the liquid below the beam. The fluid motion generates two forces: a lift, perpendicular to the blade, due to the lubrication pressure $p$ and a viscous drag force $f_{v}$, locally tangent to the surface. Both are calculated by solving the Stokes equation in the lubrication approximation (see Supplementary materials). We also include the capillary forces arising from the liquid surface deformation on the left and right parts of the wet area of the blade. They exert a small torque on the blade and, more importantly, impose the fluid pressure at $s=s_{w}$ and $s=L$ through the curvature of the liquid surface. Finally, the weight of the plate is neglected. With these conditions, the derivative of the torque balance $\Gamma=E I \frac{d \theta}{d s}$ (denoting $I$ the moment of inertia of the blade and $\theta$ the local tangent angle of the blade with respect to the vertical) writes:

$E^{*} I \frac{d^{2} \theta}{d s^{2}}=b \int_{s}^{L} f_{v} \sin \left(\theta(s)-\theta\left(s^{\prime}\right)\right)-p \cos \left(\theta(s)-\theta\left(s^{\prime}\right)\right) d s^{\prime}$

with $b$ the width of the blade. We neglect the contribution of capillary forces on the torque derivative $\frac{d \Gamma_{c}}{d s} \sim \gamma b$ (with $\gamma$ the surface tension of the liquid), which is more than 100 times smaller than the contribution of the lubricating film $\frac{d \Gamma_{v}}{d s} \sim p b l_{\mathrm{w}}$ (see Supplementary Materials).

In a second part, the free surface of the liquid deposited by the blade is calculated through the canonical Landau Levich equation (following [2]). The variables of the free surface are distinguished here from those of the blade by using an index $l$ ):

$$
\frac{d^{2} \theta_{l}}{d s^{2}}=-\frac{3 \eta}{\gamma}\left(\frac{V h_{l}-q / b}{h_{l}^{3}}\right) \sin \theta_{l}(s)
$$

with $h_{l}$ the film height, and $q$ the flow rate of the liquid in the reference frame of the blade.
Equations 1 and 2 can both be seen as 1D steady-state non-linear heat equations where $\theta$ is equivalent to temperature, with a conductivity equal to 1 and a source distribution $S(s, \theta(s))$ corresponding to the right hand side of the equations. We use this analogy to solve the set of coupled equations 1 and 2 (for the lubricated blade and for the deposited film). To do so, we look for the stationary asymptotic solution to a transient heat equation (Eq. 3) associated to Eqs. 1 and 2, where the derivative of $\theta$ versus a virtual time $\tau$ is added (the volumetric heat capacity is chosen unitary):

$$
\frac{d \theta}{d \tau}=\frac{d^{2} \theta}{d s^{2}}+S(s)
$$

Starting from an initial guess of the shape $\left(\theta(s)\right.$ and $\left.\theta_{l}(s)\right)$ and $p(s=L)$, Eq. 3 is solved numerically by finite differences using a semi-implicit scheme [19]. The non-linear source term $S(s)$ is treated explicitly while the rest is treated implicitly (see Supplementary Materials for the details). This scheme is stable, and thus ensures convergence to the stationary solution $\theta(s)$ (according to Lax equivalence theorem [20]), for a given set of input variables $l_{\mathrm{w}}, \delta_{s}$ and $\delta_{b}$. Two sets of boundary conditions complete this modeling. For the blade, the fixed mounting imposes $\theta(0)=0$, and the absence of torque at the tip gives $\left.\frac{d \theta}{d s}\right|_{s=L}=0$. For the free surface, the Laplace pressure equation imposes $\left.\frac{d \theta_{l}}{d s}\right|_{L}=-\frac{p(s=L)}{\gamma}$ at the contact of the blade tip, while far from the blade the film is horizontal, so that $\lim _{x \rightarrow+\infty} \theta_{l}=\pi / 2$. Finally, the matching of the two solutions is done iteratively by imposing a continuity of the flow rate $q$. The liquid height at $s=L$ is continuous [3], but there is an angle discontinuity, similar to what happens at a three phase contact line.

Integration of the blade and deposited film equations thus give the film thickness $e$ for a given $l_{\mathrm{w}}$, corresponding to the height of the free surface $h_{l}$ for $x \rightarrow \infty$. In Fig. , 2 , the calculated film thicknesses (black and grey dashed lines) are compared to the experiments for varying wetting lengths $l_{\mathrm{w}}$. They match the experimental data for both $\eta=480 \mathrm{mPas}$ and $\eta=960 \mathrm{mPas}$, without any adjustable parameter. In addition, the numerical solution of the blade shape (Eq. 1) also matches the experiments, as shown in the supplementary materials.

How does the wetting length influence the spreading dynamics? Since the presence of a meniscus below the blade induces a pressure jump at $s=s_{\mathrm{w}}$, a first hypothesis is that the dependency of $e$ with $l_{\mathrm{w}}$ results from an elasto-capillary competition [21, 22]. To check this idea, we compare in Fig. 3 numerical simulations of $e\left(l_{\mathrm{w}}\right)$ in two different configurations: in absence of a pressure jump in $s=s_{\mathrm{w}}$ (gray line), and for varying wetting conditions (dotted lines). A major observation from Fig. 3 is that $e$ varies with $l_{\mathrm{w}}$ even when capillary forces are absent. This indicates that the pressure jump at the 


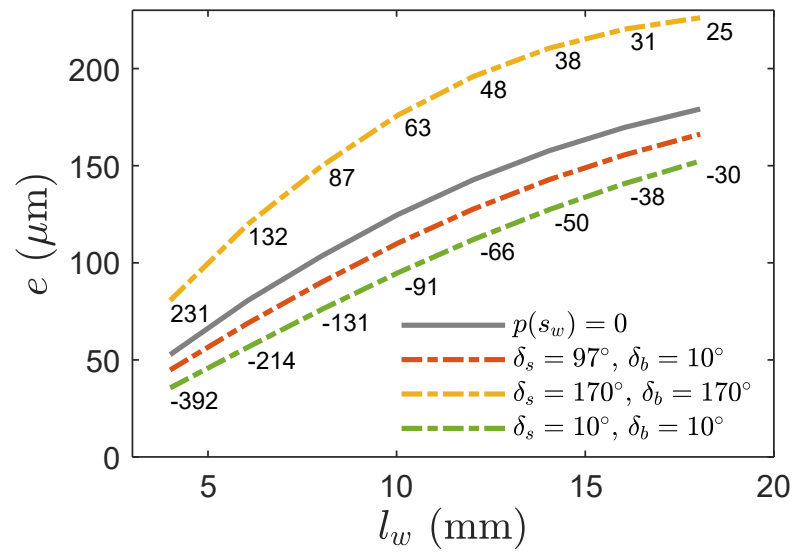

Figure 3: Effect of wetting on $e\left(l_{\mathrm{w}}\right)$. The continuous gray line corresponds to the absence of a pressure jump $p\left(s_{\mathrm{w}}\right)=0$. The green, red and yellow dotted lines show three different wetting configurations with varied contact angles $\delta_{s}$ and $\delta_{b}$ on the substrate and on the blade. The numbers indicate the pressure jump $p\left(s_{\mathrm{w}}\right)$ due to the meniscus (in $\mathrm{Pa}$ ).

meniscus does not cause the dependency of $e$ with $l_{\mathrm{w}}$. Wetting conditions only have a small influence on the film thickness, by shifting the $e\left(l_{\mathrm{w}}\right)$ curves: the dotted lines correspond to varying contact angles $\delta_{s}$ and $\delta_{b}$ on the surface and on the blade (as in Fig. 1). While $e$ is potentially increased by a factor two when comparing the almost perfect wetting $\left(\delta_{s}=\delta_{b}=10^{\circ}\right)$ to the non-wetting situation $\left(\delta_{s}=\delta_{b}=170^{\circ}\right)$, the latter is highly unlikely as the film would be unstable and dewet the substrate. In classical wetting configurations (green and red plots), capillary forces cause a modest variation of $e$, close to $20 \%$.

A second hypothesis is that the dependency of $e$ with $l_{\mathrm{w}}$ arises from a modified balance between viscous and elastic forces. Indeed, due to the finite size of the reservoir, the pressure within the sheared film applies over a variable distance $l_{\mathrm{w}}$, so that the viscous force lifting the blade diminishes as the reservoir empties. Under this assumption, we propose a scaling law for the film thickness $e$. The region of the blade wet by oil (of size $l_{\mathrm{w}}$ ) is submitted to a lubricating pressure $p \sim \eta \frac{V}{e^{2}} l_{\mathrm{w}} . p$ thus induces a torque $\Gamma_{\text {wet }}$, pushing up the wet part of the blade (of area $\left.b l_{\mathrm{w}}\right)$. The lever arm is $\sim L-l_{\mathrm{w}}$, so that $\Gamma_{\text {wet }}$ writes $\Gamma_{\text {wet }} \sim \eta \frac{V}{e^{2}} b l_{\mathrm{w}}^{2}\left(L-l_{\mathrm{w}}\right)$. At equilibrium, this torque is compensated by the rigidity of the dry part of the blade, inducing a resisting torque $\Gamma_{\text {dry }} \sim E^{*} I \frac{d \theta}{d s} \sim \frac{E^{*} I}{\left(L-l_{\mathrm{w}}\right)}$. The torque balance sets the deposition law:

$$
e \sim l_{\mathrm{w}} \sqrt{\frac{\eta V L^{2} b}{E^{*} I}}\left(1-\frac{l_{\mathrm{w}}}{L}\right)
$$

This scaling law is plotted in Fig. 2b (with a continuous line). It very convincingly reproduces the dependency of the film thickness $e$ with both $l_{\mathrm{w}}$ and $\eta$. The best fits are obtained with similar prefactors: 0.15 for $\eta=480 \mathrm{mPas}$ and 0.17 for $\eta=960 \mathrm{mPas}$.

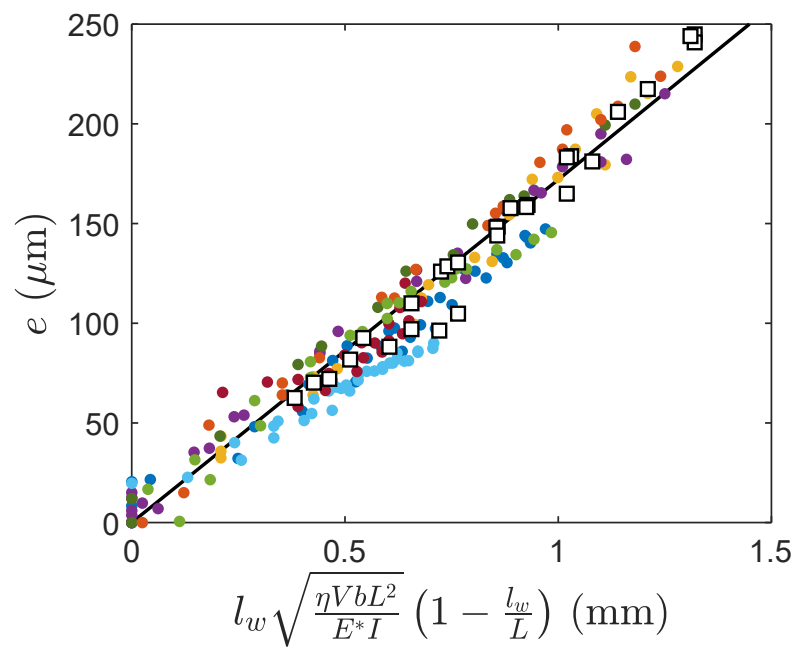

Figure 4: Experimental (colored dots) and numerical (white squares) film thickness, plotted as a function of the theoretical thickness (Eq. 4). All points collapse on a line (in black) with numerical prefactor of 0.17 . Data correspond to varying velocities $V=2.5 \mathrm{~mm} / \mathrm{s}$ (light blue), $5 \mathrm{~mm} / \mathrm{s}$ (blue, yellow, purple), $7.5 \mathrm{~mm} / \mathrm{s}$ (dark green) and $10 \mathrm{~mm} / \mathrm{s}$ (red, fuchsia pink, light green), viscosities $\eta=960$ Pa.s (purple, light green) and 480 Pa.s (all other colors), blade rigidities $B=7.1 \pm 0.5$ $\mathrm{mN} \mathrm{m}$ (light green and fuchsia pink) and $1.0 \pm 0.1 \mathrm{mN} \mathrm{m}$ (all other colors) and lengths $L=7 \mathrm{~cm}$ (yellow, fuchsia pink, light green) and $5.7 \mathrm{~cm}$ (all other colors).

To further validate our approach, we summarize in Fig. 4 the film thickness measurements for different blade rigidities $B$ and length $L$, varying liquid viscosity $\eta$ and spreading velocity $V$ (respectively varied by a factor 8 , $0.3,2$ and 4). Each color corresponds to a different set of parameters (see legend). When plotted as a function of the theoretical film thickness, all data collapse on a single line with slope 0.17 . The numerical solution of the elasto-hydrodynamic equations (Eqs. 1 and 2) is also shown with white squares. Here, the same parameters $(V, \eta, B)$ as the experiments are used, and capillarity is neglected. The numerical results also collapse on the same master curve, which is a further validation of the scaling law: it shows that the essential physical parameters are taken into account. The residual scattering of the data might be explained by the influence of capillary forces, neglected in Eq. 4, which induce a small variation of the dynamic contact angles with $V[23,24]$.

The scaling law evidences the fundamental impact of the wetting length $l_{\mathrm{w}}$, and allows us to put a central point forward. With a finite reservoir, the viscous forces of the lubricated film are exerted over an externally imposed distance $l_{\mathrm{w}}$ that varies during the spreading. This is fundamentally different from the capillary-elasticity analogy approach, where the pressure applies over an internal dynamical length $l_{\mathrm{x}} \sim\left(e L^{2}\right)^{1 / 3}[9,12]$ vary- 
ing with the blade and liquid parameters. This in turn impacts the scaling of the film thickness, which writes $e \sim L\left(\frac{\eta V b L^{2}}{E^{*} I}\right)^{3 / 4}$ in the blade-meniscus analogy [1214]. In addition, the blade is here deformed by the film over its whole length, as visible in Supplementary Figure 3 , contrary to the meniscus in dip-coating experiments. For this reason, the blade shape cannot be solved using an asymptotic matching, as usually done for a liquid interface.

This study has an important applicative scope: we indeed demonstrate that it is impossible to obtain a deposit of constant thickness with an elastic blade if the spreading is done at constant speed. In addition, we evidence the role of capillarity, which only plays a role for very hydrophobic substrates. Our work also suggests that the analogy between elastic and capillary interfaces is not valid when the blade is not fully covered with liquid.

[1] AE Hosoi and L Mahadevan. Peeling, healing, and bursting in a lubricated elastic sheet. Phys. Rev. Lett., 93(13):137802, 2004.

[2] PH Trinh, SK Wilson, and HA Stone. A pinned or freefloating rigid plate on a thin viscous film. J. Fluid Mech., 760:407-430, 2014.

[3] PH Trinh, SK Wilson, and HA Stone. An elastic plate on a thin viscous film. arXiv:1410.8558, 2014.

[4] F Saita. Simplified models of flexible blade coating. Chem. Eng. Sci., 44(4):817-825, 1989.

[5] FR Pranckh and LE Scriven. Elastohydrodynamics of blade coating. AIChE J., 36(4):587-597, 1990.

[6] CM Corvalán and FA Saita. Blade coating on a compressible substrate. Chem. Eng. Sci., 50(11):1769-1783, 1995.

[7] AJ Giacomin, JD Cook, LM Johnson, and AW Mix. Flexible blade coating. J. Coat. Technol. Res., 9(3):269-277, 2012.

[8] I Iliopoulos and LE Scriven. A blade-coating study using a finite-element simulation. Phys. Fluids, 17(12):127101, 2005.
[9] JH Snoeijer. Analogies between elastic and capillary interfaces. Phys. Rev. Fluids, 1(6):060506, 2016.

[10] L Landau and B Levich. Dragging of a liquid by a moving plate. In Dynamics of Curved Fronts, pages 141-153. Elsevier, 1988.

[11] B. Derjaguin. Thickness of liquid layer adhering to walls of vessels on their emptying and the theory of photo-and motion-picture film coating. In $C R$ (Dokl.) Acad. Sci. URSS, volume 39, pages 13-16, 1943.

[12] J Seiwert, D Quéré, and C Clanet. Flexible scraping of viscous fluids. J. Fluid Mech., 715:424-435, 2013.

[13] HN Dixit and GM Homsy. The elastic landau-levich problem. J. Fluid Mech., 732:5-28, 2013.

[14] K Warburton, DR Hewitt, and JA Neufeld. The elastic landau-levich problem on a slope. J. Fluid Mech., 883, 2020.

[15] L Mahadevan and JB Keller. Periodic folding of thin sheets. Siam Rev., 41(1):115-131, 1999.

[16] H Becker. Elastic modulus of mylar sheet. J. Appl. Polym. Sci, 9(3):911-916, 1965.

[17] SP Timoshenko and JM Gere. Theory of elastic stability. Courier Corporation, 2009.

[18] NB Rao and VG Rao. Large deflections of a cantilever beam subjected to a rotational distributed loading. Forschung im Ingenieurwesen A, 55(4):116-120, 1989.

[19] SJ Ruuth. Implicit-explicit methods for reactiondiffusion problems in pattern formation. J. Math. Biol., 34(2):148-176, 1995.

[20] PD Lax and RD Richtmyer. Survey of the stability of linear finite difference equations. Commun. Pure Appl. Math., 9(2):267-293, 1956.

[21] C Py, P Reverdy, L Doppler, J Bico, B Roman, and CN Baroud. Capillary origami: spontaneous wrapping of a droplet with an elastic sheet. Phys. Rev. Lett., 98(15):156103, 2007.

[22] José Bico, Étienne Reyssat, and Benoît Roman. Elastocapillarity: When surface tension deforms elastic solids. Annu. Rev. Fluid Mech, 50:629-659, 2018.

[23] OV Voinov. Hydrodynamics of wetting. Fluid Dyn., 11(5):714-721, 1976.

[24] RG Cox. The dynamics of the spreading of liquids on a solid surface. part 1. viscous flow. J. Fluid Mech., 168:169-194, 1986 